\title{
Graphene-Coated Elliptical Nanowires for Low Loss Subwavelength Terahertz Transmission
}

\author{
Da Teng ${ }^{1,2, *(\mathbb{D}}$, Kai Wang ${ }^{3, *}$, Zhe Li $^{4}$, Yongzhe Zhao ${ }^{1}$, Gao Zhao ${ }^{1}$, Huiyong Li $^{1}$ and \\ Heng Wang ${ }^{1}$
}

1 School of Physics and Electronic Engineering, Zhengzhou Normal University, Zhengzhou 450044, China; zyzcold@126.com (Y.Z.); zhaog1988@126.com (G.Z.); lihuiyong0528@163.com (H.L.); wh402401575@163.com (H.W.)

2 Quantum Materials Research Center, School of Physics and Electronic Engineering, Zhengzhou Normal University, Zhengzhou 450044, China

3 Key Laboratory of Infrared Imaging Materials and Detectors, Shanghai Institute of Technical Physics, Chinese Academy of Sciences, Shanghai 200083, China

4 Department of Physics, Shanghai University, 99 Shangda Road, Baoshan District, Shanghai 200444, China; lizhecn@shu.edu.cn

* Correspondence: tengda@zznu.edu.cn (D.T.); wkbenpao.optics@gmail.com (K.W.); Tel.: +86-0371-6550-2273 (D.T.); +86-021-2505-1475 (K.W.)

Received: 3 May 2019; Accepted: 3 June 2019; Published: 8 June 2019

\begin{abstract}
Graphene has been recently proposed as a promising alternative to support surface plasmons with its superior performances in terahertz and mid-infrared range. Here, we propose a graphene-coated elliptical nanowire (GCENW) structure for subwavelength terahertz waveguiding. The mode properties and their dependence on frequency, nanowire size, permittivity and chemical potential of graphene are studied in detail by using a finite element method, they are also compared with the graphene-coated circular nanowires (GCCNWs). Results showed that the ratio of the long and short axes $(b / a)$ of the elliptical nanowire had significant influence on mode properties, they also showed that a propagation length over $200 \mu \mathrm{m}$ and a normalized mode area of approximately $10^{-4} \sim 10^{-3}$ could be obtained. Increasing $b / a$ could simultaneously achieve both long propagation length and very small full width at half maximum (FWHM) of the focal spots. When $b / a=10$, a pair of focal spots about $40 \mathrm{~nm}$ could be obtained. Results also showed that the GCENW had a better waveguiding performance when compared with the corresponding GCCNWs. The manipulation of Terahertz (THz) waves at a subwavelength scale using graphene plasmon (GP) may lead to applications in tunable $\mathrm{THz}$ components, imaging, and nanophotonics.
\end{abstract}

Keywords: Terahertz wave; graphene plasmon; subwavelength structure; nanophotonics

\section{Introduction}

Terahertz (THz) waves, located between millimeter radio waves and far infrared waves, have been widely investigated in the fields of spectroscopy, imaging, and communications [1-4]. In particular, the $\mathrm{THz}$ waveguide has become a hot research topic, and semiconductor based plasmonic waveguides [5], pure dielectric waveguides [6-8], and noble metal plasmon waveguides [9-20] have been proposed to guide $\mathrm{THz}$ waves. However, in the $\mathrm{THz}$ range, surface plasmon effects are very weak, which leads to relatively poor confinement, thus hindering the applications in the subwavelength scale. Although the strong mode confinement could be achieved, it usually results in a limited propagation length [21,22].

Recent reports show that graphene [23] can also support plasmons [24,25], which provides an alternative approach to transmitting $\mathrm{THz}$ waves [26]. Compared with the metal plasmon waveguides, GP waveguides show very strong modal field confinement, and the chemical potential of graphene 
could be tuned to improve the waveguiding performance [25]. So far, graphene sheet [27], graphene gap waveguide [28], V-shaped graphene waveguide [29], graphene-based parallel-plate waveguide [30], dielectric loaded graphene waveguide [31], graphene hybrid waveguides [32-36], and graphene-coated dielectric nanowires [37-44] have been proposed and investigated. Among them, graphene hybrid waveguides have shown very good waveguiding performances in the THz band. Zhou et al. [32] proposed a graphene hybrid plasmonic waveguide with a propagation distance of approximately $127 \mu \mathrm{m}$, and a normalized mode area of approximately $10^{-2}$ at $3 \mathrm{THz}$. Later, a symmetric hybrid plasmonic waveguide [33] was proposed to achieve a propagation length of $26.7 \mathrm{~mm}$ at $10 \mathrm{THz}$, while the normalized mode area is still around $10^{-2}$. To further downscale the mode area, He et al. [34,35] proposed two graphene-based hybrid plasmonic waveguides, which could simultaneously achieve a very small normalized modal area of approximately $10^{-4} \sim 10^{-3}$ and a propagation length of approximately several hundreds of micrometers at $3 \mathrm{THz}$. Recently, graphene-coated nanowires have attracted lots of research interest and been investigated mainly in the mid-infrared band [37-44]. In the THz band, a graphene-coated nanowire with a drop-shaped cross section was suggested for low loss waveguiding with ultra-strong mode confinement [44]. Although the propagation distance has been enhanced to millimeter range, these structures are relatively complicated for practical implementations.

Here, we present that a simple graphene-coated elliptical nanowire (GCENW) could simultaneously achieve a very small normalized modal area of approximately $10^{-4} \sim 10^{-3}$ and a propagation length of several hundreds of micrometers. Meanwhile, in addition to the simple structure, the GCENW could provide two focal spots with the same size. Results show that the ratio of $b / a$ has significant influence on mode properties, increasing $b / a$ could simultaneously achieve both a long propagation length and a very small full width at half maximum (FWHM) of the focal spots. By adjusting the chemical potential of graphene, the waveguiding properties could also be tuned to achieve better performance.

\section{Theoretical Model and Methods}

The permittivity of graphene can be obtained by using $\varepsilon_{\mathrm{g}}=1+i \sigma_{\mathrm{g}} /\left(\varepsilon_{0} \omega \Delta\right)[25,45]$, where $\Delta=0.33 \mathrm{~nm}$ is the thickness of the graphene layer, $\omega$ is the frequency, $\varepsilon_{0}$ is the permittivity in free space, and $\sigma_{\mathrm{g}}$ is the surface conductivity of graphene. Under the random-phase approximation, the dynamic optical response of graphene can be obtained from Kubo's formula [45-47]. In the terahertz range, the intraband transition of electrons dominates [48,49], and then $\sigma_{\mathrm{g}}$ could be approximated as

$$
\sigma_{g}=\frac{2 i e^{2} k_{B} T}{\pi \hbar^{2}(\omega+i / \tau)} \ln \left[2 \cosh \left(\frac{u_{c}}{2 k_{B} T}\right)\right]
$$

where $\tau=0.5 \mathrm{ps}, T=300 \mathrm{~K}, u_{\mathrm{c}}$ is the chemical potential, $\hbar$ is the reduced Plank's constant, $k_{B}$ is the Boltzmann constant, and $e=1.6 \times 10^{-19} \mathrm{C}$, and $\varepsilon_{2}=1$.

For the proposed scheme shown in Figure 1a, we assume that the lowest order graphene plasmon (GP) mode transmits along $z$-direction with a time dependence of $e^{-\mathrm{j} \omega t}$. Then the transversal mode fields (E and H) satisfy a two-dimensional wave equation [20],

$$
\left[\nabla_{t}^{2}+k_{0}^{2}\left(\varepsilon \mu-n_{e f f}^{2}\right)\right]\left\{\begin{array}{c}
E(x, y) \\
H(x, y)
\end{array}\right\}=0
$$

where $\varepsilon$ and $\mu$ are the permittivity and permeability. Since the eigenvalue equation here is very complicated, we adopt the finite element method software COMSOL Multiphysics to calculate the complex effective mode index, $n_{\text {eff }} \beta=k_{0} n_{\text {eff }}$ is the complex propagation constant with $k_{0}=2 \pi / \lambda_{0}$, where $\lambda_{0}$ is the wavelength in the free space. The propagation length is defined as $L_{\mathrm{P}}=1 / \operatorname{Im}(\beta)$. The normalized mode area is defined as $A_{\text {eff }} / A_{0}$, where $A_{0}=\lambda^{2}{ }_{0} / 4$ is the diffraction-limited mode area, and the effective mode area $A_{\text {eff }}$ is obtained by [50-52], 


$$
A_{\mathrm{eff}}=\frac{\iint W(\mathrm{r}) d^{2} \mathrm{r}}{\max \{W(\mathrm{r})\}},
$$

where $W(r)$ is the electromagnetic energy density and is as follows:

$$
W(\mathrm{r})=\frac{1}{2}\left\{\frac{d[\varepsilon(\mathrm{r}) \omega]}{d \omega}|\mathrm{E}(\mathrm{r})|^{2}+\mu_{0}|\mathrm{H}(\mathrm{r})|^{2}\right\} .
$$

We also adopted a figure of merit $(F o M)[39,53]$ to assess the propagation length and effective mode area, which is defined as $F o M=\operatorname{Re}(\beta) / \operatorname{Im}(\beta)$.

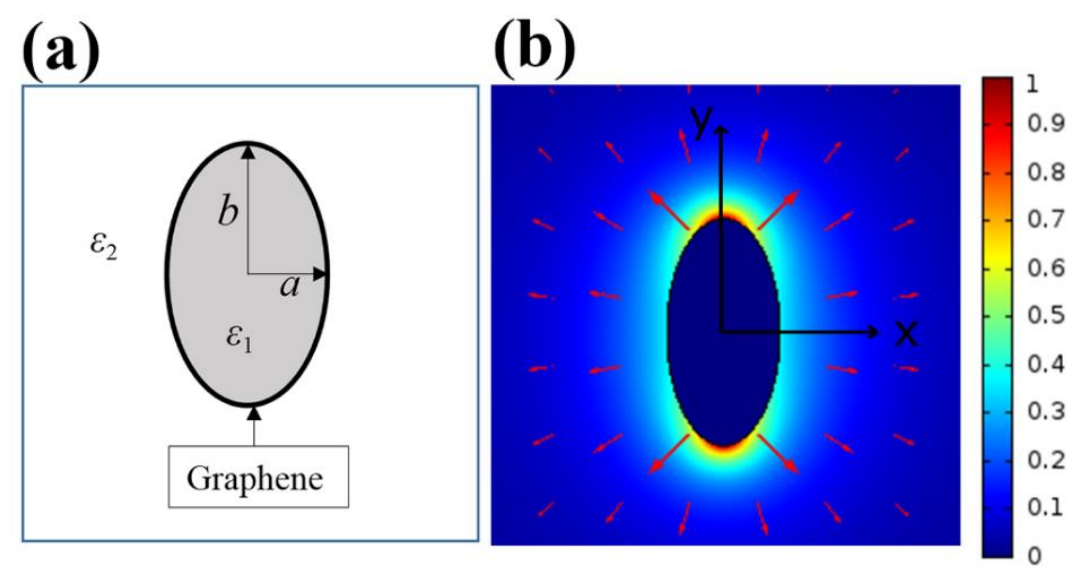

Figure 1. (a) The cross section of the graphene-coated elliptical nanowire (GCENW) waveguide. (b) Normalized electromagnetic energy distribution of the graphene plasmon (GP) mode at $3 \mathrm{THz}$. Red arrows indicate the polarization directions. The short axis and long axis are $a=0.5 \mu \mathrm{m}$ and $b=1 \mu \mathrm{m}$, respectively. The other parameters are $u_{\mathrm{c}}=0.5 \mathrm{eV}, \varepsilon_{1}=2.25$, and $\varepsilon_{2}=1$.

\section{Results}

We considered the GCENW waveguide to be surrounded by a dielectric medium $\left(\varepsilon_{2}\right)$. The elliptical dielectric nanowire $\left(\varepsilon_{1}\right)$ was covered by monolayer graphene. As shown in Figure 1a, the short axis and long axis were $a$ and $b$. Here we placed emphasis on the lowest order mode for its long propagation distance. Figure $1 \mathrm{~b}$ shows the energy distribution of the lowest order GP mode for $a=0.5 \mu \mathrm{m}$ and $b=1 \mu \mathrm{m}$ at $3 \mathrm{THz}$, the majority of the optical energy was restricted in the tips, which is more evident for a larger $b / a$. The Poynting vector in $z$ direction $\left(S_{z}\right)$ had the largest value at the surface and decayed rapidly away from the interface, seen in Figure 2. Since the elliptical nanowire has two tips, two focal spots with same size could be obtained. Figure $2 \mathrm{a}$, and $2 \mathrm{~b}$ present the FWHM [51] of $S_{z}$ at the tips for $a=0.5 \mu \mathrm{m}, b=1 \mu \mathrm{m}$ and $a=0.5 \mu \mathrm{m}, b=5 \mu \mathrm{m}$, which were about $140 \mathrm{~nm}$ and $40 \mathrm{~nm}$, respectively. Surprisingly, when increasing $b / a$ from 2 to 10, the FWHM of the focal spots reduced from $140 \mathrm{~nm}$ to $40 \mathrm{~nm}$, and the propagation length increased from $83 \mu \mathrm{m}$ to $136 \mu \mathrm{m}$. Hence, the ratio of $b / a$ had significant influence on the spot size and propagation length. As shown in Table 1 , increasing $b / a$, the FWHM could be further reduced. However, when $b / a$ increased to a certain value, the elliptical nanowire gradually becomes an approximate parallel plate. Therefore, the ratio of $b / a$ should not be much larger. 


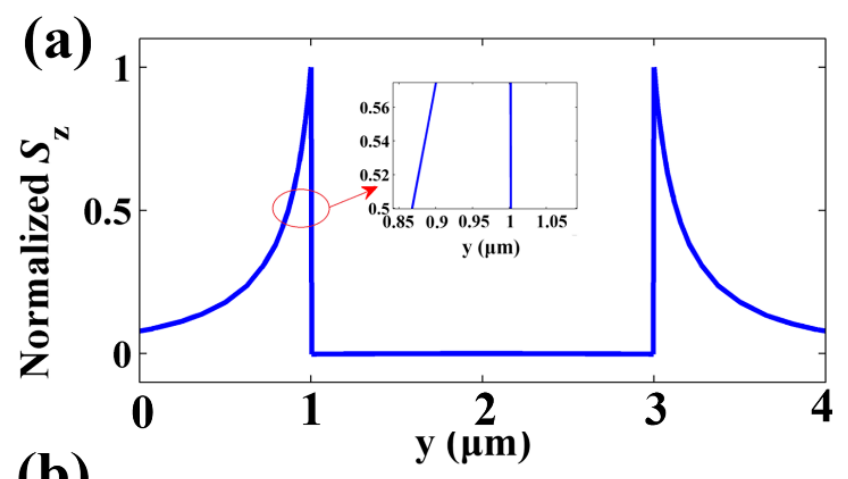

(b)

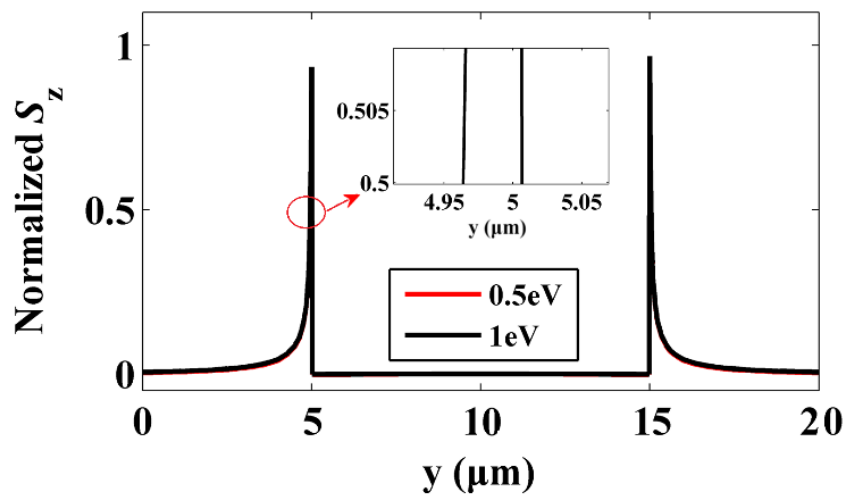

Figure 2. The time-averaged Poynting vector $S_{\mathrm{z}}$ along $y$ axis for $(\mathbf{a}) b / a=2, u_{\mathrm{c}}=0.5 \mathrm{eV}$, and (b) $b / a=10$, $u_{\mathrm{c}}=0.5 \mathrm{eV}, 1 \mathrm{eV}$ at $3 \mathrm{THz}$. The center coordinates of the ellipses are $(0,2 \mu \mathrm{m})$ and $(0,10 \mu \mathrm{m})$. Here, $a=0.5 \mu \mathrm{m}, \varepsilon_{1}=2.25$, and $\varepsilon_{2}=1$. The insets show the enlarged detail of $S_{\mathrm{z}}$.

Table 1. The mode properties versus $b / a$, and $a=0.5 \mu \mathrm{m}, u_{\mathrm{c}}=0.5 \mathrm{eV}, \varepsilon_{1}=2.25$, and $\varepsilon_{2}=1$.

\begin{tabular}{cccc}
\hline $\boldsymbol{b} / \boldsymbol{a}$ & $\boldsymbol{n}_{\text {eff }}$ & $\boldsymbol{L}_{\mathbf{P}}(\boldsymbol{\mu m})$ & FWHM $(\mathbf{n m})$ \\
\hline 2 & $3.0709+0.19168 i$ & 83 & 140 \\
3 & $2.7599+0.17064 i$ & 93 & 100 \\
5 & $2.4004+0.14512 i$ & 110 & 75 \\
10 & $2.0191+0.11677 i$ & 136 & 40 \\
15 & $1.8561+0.10439 i$ & 152 & 36 \\
20 & $1.7646+0.09751 i$ & 163 & 32 \\
\hline
\end{tabular}

Figure 3a demonstrates the dependencies of the effective mode index and propagation length on frequency for the GP mode. As the frequency ranges from $1 \mathrm{THz}$ to $5 \mathrm{THz}, \operatorname{Re}\left(n_{\mathrm{eff}}\right)=\operatorname{Re}(\beta) / k_{0}$ gradually increased, while the propagation length $L_{P}$ decreased with frequency increasing. At higher frequencies, the much larger absorption of graphene led to the enhancement of the propagation loss. Figure $3 \mathrm{~b}$ shows the normalized mode area $\left(A_{\text {eff }} / A_{0}\right)$, and FoM of the GP mode as a function frequency. When $f_{0}=1 \mathrm{THz}$ and $b / a=2, L_{\mathrm{P}}$ is approximately $125 \mu \mathrm{m}$, and $A_{\text {eff }} / A_{0}$ is only $2.44 \times 10^{-4}$. When $b / a=3, L_{\mathrm{P}}$ is approximately $146 \mu \mathrm{m}$, i.e., increased by $16.8 \%$, while the normalized mode area became slightly smaller, seen in Figure 3b. As a result, the GCENW with a larger b/a showed better performance both in propagation length and mode area.

We further discuss the impact of the ratio of $b / a$ on the modal behavior in GCENW. Here, it is necessary to clarify that one can keep $a$ or $b$ constant while changing another. Thus, we set $a=1 \mu \mathrm{m}$ and swept $b$ from $0.5 \mu \mathrm{m}$ to $3 \mu \mathrm{m}$ (i. e., $b / a$ ranging from 0.5 to 3 ) at $3 \mathrm{THz}$. Figure 4 a depicts $\operatorname{Re}\left(n_{\text {eff }}\right)$ and $L_{\mathrm{P}}$ with respect to $b$, and one can see that $\operatorname{Re}\left(n_{\mathrm{eff}}\right)$ decreased with the increase of $b$. For the lowest order mode, propagation length increased with $b$ increasing, and $L_{\mathrm{P}}$ is about $83 \mu \mathrm{m}$ and $107.7 \mu \mathrm{m}$ for $b / a=0.5$ and $b / a=3$, respectively. As for the normalized mode area shown in Figure $4 \mathrm{~b}$, there was a maximum value at $b / a=1$, which is depicted by the vertical black dotted line. This result implies that 
the graphene-coated circular nanowire (GCCNW) is not the optimal choice. The GP mode in GCENW had superior performances ( $b / a$ ranging from 1 to 3 ) both in propagation length and mode area when compared with the GCCNW $(b / a=1)$. We will discuss this in Section 4 . In addition, the FoM had a minimum value of $b / a=1.5$. Therefore, in order to achieve a longer propagation length and smaller mode area, larger $b / a$ values are preferred. The results are in consistent with that of Figure 2.
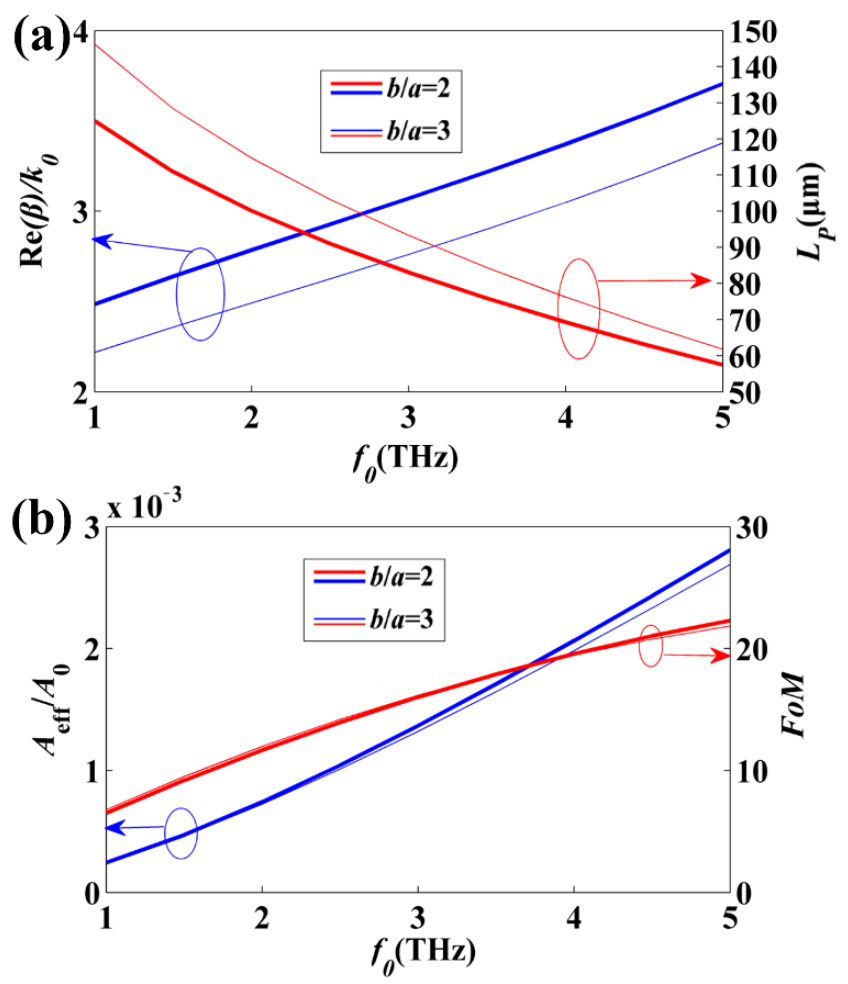

Figure 3. (a) Real part of $n_{\mathrm{eff}}$ and $L_{\mathrm{P}}$, and (b) $A_{\mathrm{eff}} / A_{0}$, and figure of merit (FoM) of the GP mode as a function of frequency. Here $u_{\mathrm{c}}=0.5 \mathrm{eV}$, and $a=0.5 \mu \mathrm{m}$. The thick and thin lines stand for $b / a=2$ and $b / a=3$, respectively.
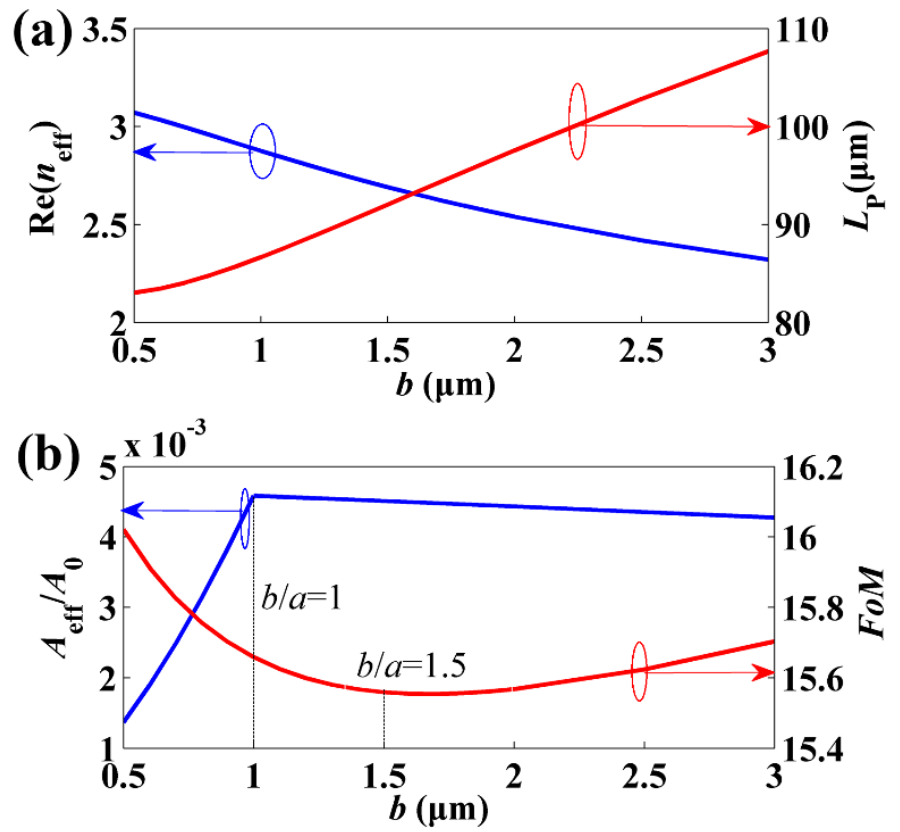

Figure 4. (a) Real part of $n_{\mathrm{eff}}$ and $L_{\mathrm{P}}$, and (b) $A_{\mathrm{eff}} / A_{0}$ and FoM as a function of $b$ at $f_{0}=3 \mathrm{THz}$. Here $u_{\mathrm{c}}=0.5 \mathrm{eV}, \varepsilon_{1}=2.25, \varepsilon_{2}=1$, and $a=1 \mu \mathrm{m}$. 
The permittivity of nanowire provided another degree of freedom to be adjusted, which also had a great influence on GP mode in GCENW. Figure 5 a shows the relationship between $\operatorname{Re}\left(n_{\mathrm{eff}}\right), L_{\mathrm{P}}$ and the permittivity at $3 \mathrm{THz}$. With increasing $\varepsilon_{1}, \operatorname{Re}\left(n_{\text {eff }}\right)$ almost linearly increased as well as the loss (i.e., decrease of $L_{\mathrm{P}}$ ). Figure $5 \mathrm{~b}$ presents $A_{\mathrm{eff}} / A_{0}$ and $F o M$ with respect to nanowire permittivity. The change of $A_{\text {eff }} / A_{0}$ with increasing permittivity was very small. For $\varepsilon_{1}=10$ and $\varepsilon_{1}=2$, the relative error of the normalized mode area was less than $10 \%$. Thus, the permittivity seemed to have a very slight impact on $A_{\text {eff }} / A_{0}$. Therefore, smaller dielectric permittivity results in better performance of the GP mode.
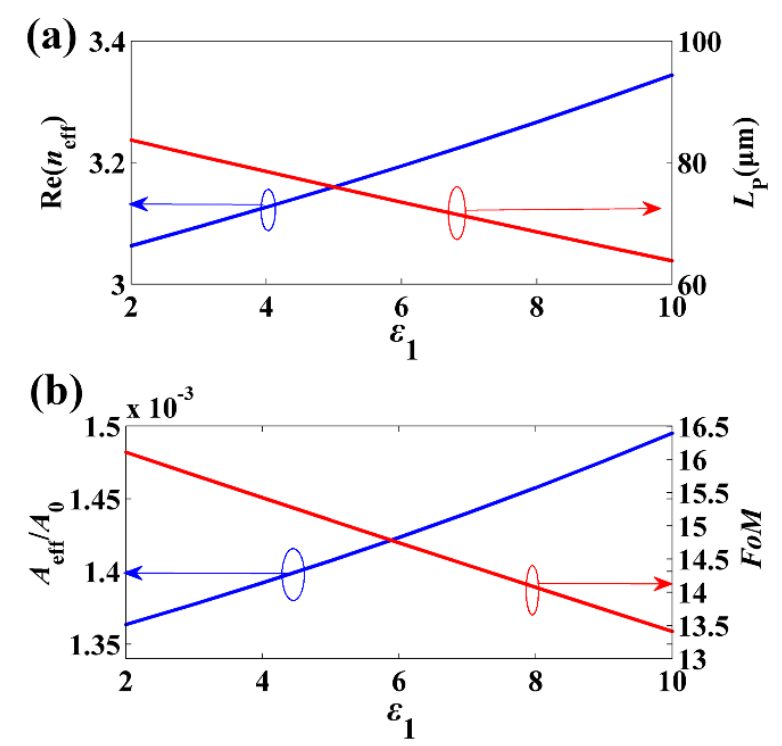

Figure 5. (a) Real part of $n_{\text {eff }}$ and $L_{\mathrm{P}}$, and (b) $A_{\text {eff }} A_{0}$ and $F o M$ as a function of nanowire permittivity at $f_{0}=3 \mathrm{THz}$. Here $u_{\mathrm{c}}=0.5 \mathrm{eV}, a=0.5 \mu \mathrm{m}, b=1 \mu \mathrm{m}$, and $\varepsilon_{2}=1$.

The surface conductivity $\sigma_{\mathrm{g}}$ of graphene could be adjusted by changing chemical potential $u_{\mathrm{c}}$. Figure 6 shows the dependences of the GP mode on the chemical potential at $f_{0}=3 \mathrm{THz}$. When the chemical potential increased from $0.2 \mathrm{eV}$ to $1 \mathrm{eV}$, the effective mode index gradually decreased, while $L_{\mathrm{P}}$ linearly increased, as depicted in Figure 6a. The normalized mode area was enlarged by less than 2-fold when $u_{\mathrm{c}}$ ranging from $0.2 \mathrm{eV}$ to $1 \mathrm{eV}$, shown in Figure $6 \mathrm{~b}$. For $b / a=2$ and $u_{\mathrm{c}}=1 \mathrm{eV}, L_{\mathrm{P}}$ and $A_{\text {eff } /} A_{0}$ are $147 \mu \mathrm{m}$ and $1.6 \times 10^{-3} A_{0}$, respectively. When further increasing $b / a$ to 10 , the effective mode index of the GP mode was $n_{\text {eff }}=1.4792+0.055868 \mathrm{i}$. The corresponding $L_{\mathrm{P}}$ and $A_{\text {eff }} / A_{0}$ were about $285 \mu \mathrm{m}$ and $1.4 \times 10^{-3}$.

As shown in Figure $2 \mathrm{~b}$, it appeared that when increasing $u_{\mathrm{c}}$ from $0.5 \mathrm{eV}$ to $1 \mathrm{eV}$, the normalized $S_{\mathrm{z}}$ was overlapped with that of $u_{\mathrm{c}}=0.5 \mathrm{eV}$. Thus, the FWHM of the focal spots was still $40 \mathrm{~nm}$. Finally, the increase of $u_{\mathrm{c}}$ resulted in the enlargement of FoM. These outcomes imply the possibility of achieving a superior performance of the GCENW by enhancing $u_{\mathrm{c}}$ and $b / a$. 

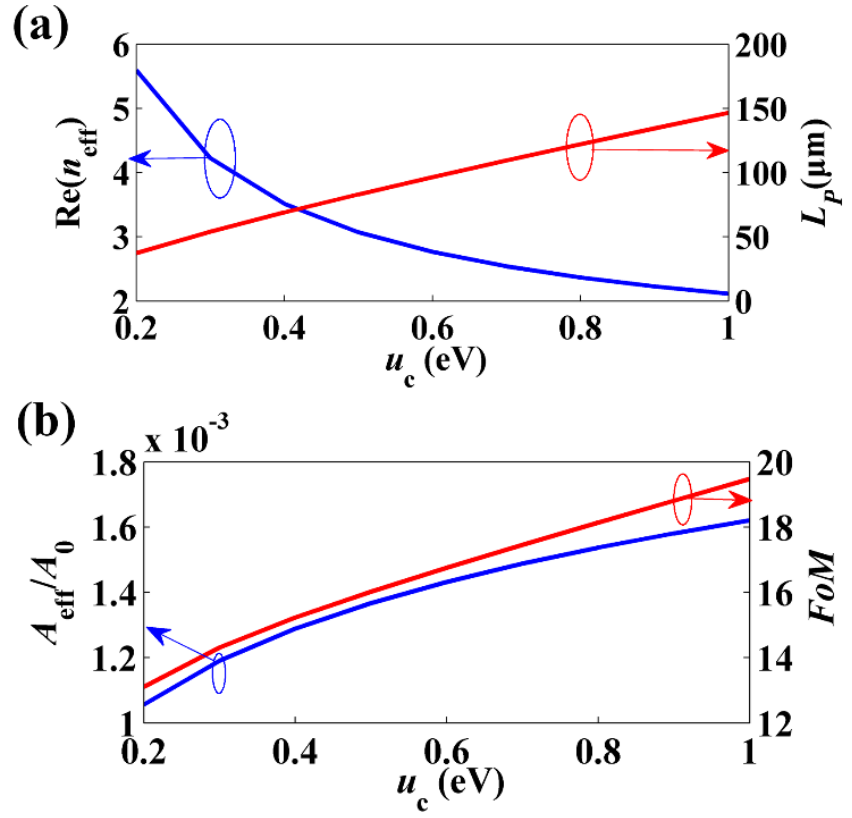

Figure 6. (a) Real part of $n_{\mathrm{eff}}$ and $L_{\mathrm{P}}$, and (b) $A_{\text {eff }} A_{0}$ and $F o M$ as a function of chemical potential $u_{\mathrm{c}}$ at $f_{0}=3 \mathrm{THz}$. Here $a=0.5 \mu \mathrm{m}, b / a=2, \varepsilon_{1}=2.25$, and $\varepsilon_{2}=1$.

\section{Discussion}

In Figure 4, we studied the dependences of mode properties on the ratio of $b / a$. Here, we compared the GCENW with two GCCNW waveguides with radii equal to $a$ and $b$. Figure $7 \mathrm{a}-\mathrm{c}$ shows the normalized energy distributions of the GP modes for graphene-coated nanowire with $R=a=1 \mu \mathrm{m}$, GCENW with $a=1 \mu \mathrm{m}$ and $b=3 \mu \mathrm{m}$, and graphene-coated nanowire with $R=b=3 \mu \mathrm{m}$, respectively. In Figure $7 \mathrm{~d}$ we compared the propagation length of these three GP modes. The red line shows the propagation length of the GP mode in GCENW, with $a=1 \mu \mathrm{m}$ and $b / a$ ranging from 1 to 3 . The gold and blue lines show the propagation length for the plasmon modes of Figure $7 \mathrm{a}$, and $7 \mathrm{c}$, which are $L_{\mathrm{P}}=86.6 \mu \mathrm{m}$ and $L_{\mathrm{P}}=95.7 \mu \mathrm{m}$, respectively. Therefore, the GP mode in GCENW had a longer propagation length for $b / a>1.8$ when comparing with GCCNW plasmon modes (with $R=1 \mu \mathrm{m}$ and $R=3 \mu \mathrm{m})$.

Meanwhile, we already knew that the effective mode area of the GP mode was smaller than that of the GCCNW plasmon mode with $R=a=1 \mu \mathrm{m}$, seen in Figure $4 \mathrm{~b}$. As for the GCCNW plasmon mode with $R=b=3 \mu \mathrm{m}$, the effective mode area was about $2.66 \times 10^{-2} A_{0}$, which is enlarged by one order of magnitude when compared with the case of $R=a=1 \mu \mathrm{m}$.

Furthermore, we obtained that the FWHM of $S_{z}$ at the tip was $250 \mathrm{~nm}$ for $b=2 \mu \mathrm{m}$ and $a=1 \mu \mathrm{m}$ ( $140 \mathrm{~nm}$ for $b=1 \mu \mathrm{m}$ and $a=0.5 \mu \mathrm{m}$, seen in Figure 2a). For GCCNW plasmon mode with $R=1 \mu \mathrm{m}$ and $R=3 \mu \mathrm{m}$, the FWHMs of $S_{z}$ were approximately $400 \mathrm{~nm}$ and $1000 \mathrm{~nm}$, respectively. As the field outside the circular wire was radially polarized, the circular wire shows relatively weak mode confinement. Additionally, the whole graphene layer led to large loss. As for GCENW, the nanofocusing effect of the elliptical nanowire tip resulted in strong modal field confinement, just as the conical metal nanowires. At the same time, only a small fraction of graphene contributed to loss, thus the propagation loss was reduced when compared with circular wire. These results indicated that the plasmon modes in GCCNW were less confined when compared to that of the GCENW, and provided a reasonable explanation for the smaller effective mode area of the GP mode in GCENW. 


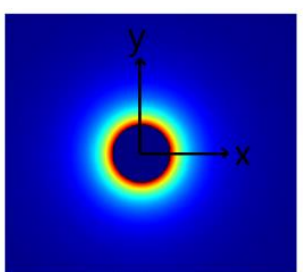

(a)

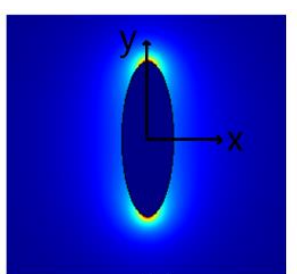

(b)

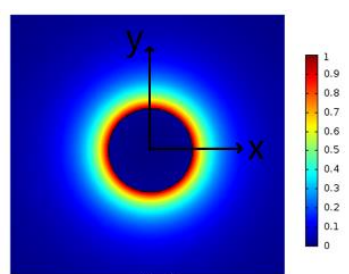

(c)

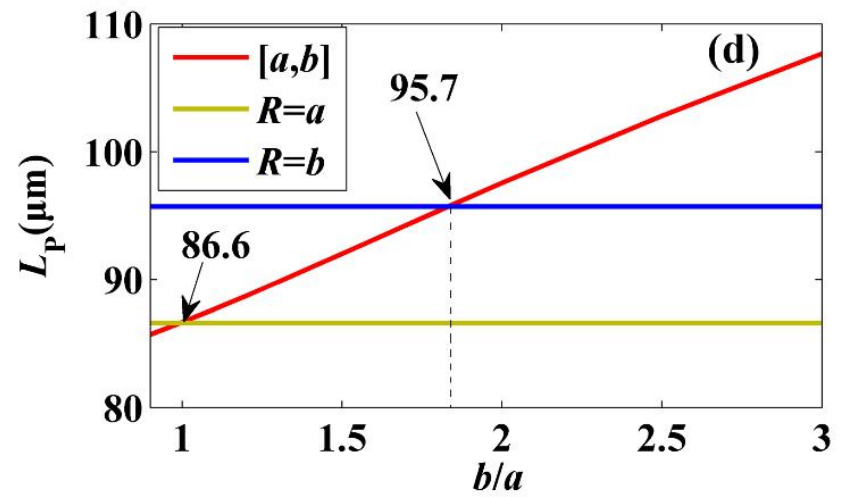

Figure 7. Normalized energy distributions for three GP modes. (a) $R=a=1 \mu \mathrm{m}$, (b) $a=1 \mu \mathrm{m}$ and $b=3$ $\mu \mathrm{m},(\mathbf{c}) R=b=3 \mu \mathrm{m}$, (d) comparison of the propagation length. The other parameters are $u_{\mathrm{c}}=0.5 \mathrm{eV}$, $\varepsilon_{1}=2.25, \varepsilon_{2}=1, a=1 \mu \mathrm{m}$, and $f_{0}=3 \mathrm{THz}$.

\section{Conclusions}

In conclusion, we investigated the subwavelength waveguiding properties of the graphene-coated elliptical nanowires for $\mathrm{THz}$ waves. A propagation distance of $285 \mu \mathrm{m}$ and an effective mode area of $10^{-3} \mathrm{~A}_{0}$ or smaller were obtained. Results showed that larger $b / a$, smaller nanowire permittivity, and larger $u_{\mathrm{c}}$ could improve the performance of the GCENW. The GP mode in GCENW had superior subwavelength waveguiding performances, both in propagation length and mode area when compared with the corresponding GCCNWs. The introduction of graphene plasmon to guide $\mathrm{THz}$ waves far beyond the diffraction limit may have applications in integrated $\mathrm{THz}$ photonic devices and $\mathrm{THz}$ imaging.

Author Contributions: D.T. and K.W. designed the waveguide structure, and helped proceeding the simulation processes and data analysis; Z.L. organized the paper and contributed in paper writing; Y.Z., G.Z., H.L. and H.W. contributed in paper writing.

Funding: This research was funded partly by the open research Fund of Zhengzhou Normal University, the aid program for Science and Technology Innovative Research Team of Zhengzhou Normal University.

Conflicts of Interest: The authors declare no conflicts of interest.

\section{References}

1. Exter, M.; Fattinger, C.; Grischkowsky, D. Terahertz time-domain spectroscopy of water vapor. Opt. Lett. 1989, 14, 1128-1130. [CrossRef] [PubMed]

2. Hu, B.B.; Nuss, M.C. Imaging with terahertz waves. Opt. Lett. 1995, 20, 1716-1718. [CrossRef] [PubMed]

3. Mittleman, D.M.; Jacobsen, R.H.; Nuss, M.C. T-ray Imaging. IEEE J. Sel. Top. Quantum Electron. 1997, 2, 679-692. [CrossRef]

4. Fitch, M.J.; Osiander, R. Terahertz waves for communications and sensing. Johns Hopkins APL Tech. Dig. 2004, 25, 348-355.

5. Li, S.; Jadidi, M.M.; Murphy, T.E.; Kumar, G. Terahertz surface plasmon polaritons on a semiconductor surface structured with periodic V-grooves. Opt. Express 2013, 21, 7041-7049. [CrossRef] [PubMed]

6. Ung, B.; Mazhorova, A.; Dupuis, A.; Rozé, M.; Skorobogatiy, M. Polymer microstructured optical fibers for terahertz wave guiding. Opt. Express 2011, 19, B848-B861. [CrossRef] [PubMed]

7. Markov, A.; Guerboukha, H.; Skorobogatiy, M. Hybrid metal wire-dielectric terahertz waveguides: Challenges and opportunities [Invited]. J. Opt. Soc. Am. B 2014, 31, 2587. [CrossRef] 
8. Atakaramians, S.; Afshar, S.; Monro, T.M.; Abbott, D. Terahertz dielectric waveguides. Adv. Opt. Photonics 2013, 5, 169-215. [CrossRef]

9. Wang, J.; Du, J. Plasmonic and dielectric metasurfaces: Design, fabrication and applications. Appl. Sci. 2016, 6, 239. [CrossRef]

10. Wang, K.; Mittleman, D.M. Metal wires for terahertz wave guiding. Nature 2004, 432, 376-379. [CrossRef]

11. Cao, Q.; Jahns, J. Azimuthally polarized surface plasmons as effective terahertz waveguides. Opt. Express 2005, 13, 511-518. [CrossRef] [PubMed]

12. Xu, L.; Li, F.; Wei, L.; Zhou, J.; Liu, S. Design of surface plasmon nanolaser based on MoS 2. Appl. Sci. 2018, 8, 2110. [CrossRef]

13. Heshmat, B.B.; Li, D.; Darcie, T.E.; Gordon, R. Tuning plasmonic resonances of an annular aperture in metal plate. Opt. Express 2011, 19, 5912-5923. [CrossRef] [PubMed]

14. Maier, S.A.; Andrews, S.R.; Martín-Moreno, L.; García-Vidal, F.J. Terahertz surface plasmon-polariton propagation and focusing on periodically corrugated metal wires. Phys. Rev. Lett. 2006, 97, 176805. [CrossRef] [PubMed]

15. Teng, D.; Cao, Q.; Gao, H.; Wang, K.; Zhu, M. Three-wave approximation for the modal field inside high-index dielectric rods of hybrid plasmonic waveguides. J. Mod. Opt. 2016, 63, 1451-1456. [CrossRef]

16. Liang, H.; Ruan, S.; Zhang, M. Terahertz surface wave propagation and focusing on conical metal wires. Opt. Express 2008, 16, 18241. [CrossRef]

17. Zheng, Z.; Kanda, N.; Konishi, K.; Kuwata-Gonokami, M. Efficient coupling of propagating broadband terahertz radial beams to metal wires. Opt. Express 2013, 21, 10642-10650. [CrossRef]

18. Mbonye, M.; Mendis, R.; Mittleman, D.M. A terahertz two-wire waveguide with low bending loss. Appl. Phys. Lett. 2009, 95, 233506. [CrossRef]

19. Pahlevaninezhad, H.; Darcie, T.E.; Heshmat, B. Two-wire waveguide for terahertz. Opt. Express 2010, 18, 7415-7420. [CrossRef]

20. Teng, D.; Cao, Q.; Li, S.; Gao, H. Tapered dual elliptical plasmon waveguides as highly efficient terahertz connectors between approximate plate waveguides and two-wire waveguides. J. Opt. Soc. Am. A 2014, 31, 268-273. [CrossRef]

21. Liang, H.; Ruan, S.; Zhang, M.; Su, H. Nanofocusing of terahertz wave on conical metal wire waveguides. Opt. Commun. 2010, 283, 262-264. [CrossRef]

22. He, X. Investigation of terahertz surface waves of a metallic nanowire. J. Opt. Soc. Am. B 2010, $27,2298$. [CrossRef]

23. Bonaccorso, F.; Sun, Z.; Hasan, T.; Ferrari, A.C. Graphene photonics and optoelectronics. Nat. Photonics 2010, 4, 611. [CrossRef]

24. Politano, A.; Chiarello, G. Plasmon modes in graphene: Status and prospect. Nanoscale 2014, 6, 10927-10940. [CrossRef] [PubMed]

25. Vakil, A.; Engheta, N. Transformation optics using graphene. Science 2011, 332, 1291-1294. [CrossRef] [PubMed]

26. Jablan, M.; Buljan, H.; Soljacic, M. Plasmonics in graphene at infrared frequencies. Phys. Rev. B 2009, 80, 245435. [CrossRef]

27. Wang, B.; Zhang, X.; Garcia-Vidal, F.J.; Yuan, X.; Teng, J. Strong coupling of surface plasmon polaritons in monolayer graphene sheet arrays. Phys. Rev. Lett. 2012, 109, 073901. [CrossRef]

28. Ding, Y.; Guan, X.; Zhu, X.; Hu, H.; Bozhevolnyi, S.I.; Oxenlowe, L.K.; Jin, K.J.; Mortensen, N.A.; Xiao, S. Efficient electro-optic modulation in low-loss graphene-plasmonic slot waveguides. Nanoscale 2017, 9, 15576-15581. [CrossRef]

29. Liu, P.; Zhang, X.; Ma, Z.; Cai, W.; Wang, L.; Xu, J. Surface plasmon modes in graphene wedge and groove waveguides. Opt. Express 2013, 21, 32432-32440. [CrossRef]

30. Lorestaniweiss, Z.; Salar, B. Tunable transverse-electric surface plasmon polariton waves in a parallel-plate graphene-based waveguide. Phys. Scr. 2019. accepted. [CrossRef]

31. Xu, W.; Zhu, Z.; Liu, K.; Zhang, J.; Yuan, X.; Lu, Q.; Qin, S. Dielectric loaded graphene plasmon waveguide. Opt. Express 2015, 23, 5147-5153. [CrossRef] [PubMed]

32. Zhou, X.; Zhang, T.; Chen, L.; Hong, W.; Li, X. A graphene-based hybrid plasmonic waveguide with ultra-deep subwavelength confinement. J. Lightwave Technol. 2014, 32, 3597-3601. 
33. Chen, M.; Sheng, P.; Sun, W.; Cai, J. A symmetric terahertz graphene-based hybrid plasmonic waveguide. Opt. Commun. 2016, 376, 41-46. [CrossRef]

34. He, X.; Ning, T.; Lu, S.; Zheng, J.; Li, J.; Li, R.; Pei, L. Ultralow loss graphene-based hybrid plasmonic waveguide with deep-subwavelength confinement. Opt. Express 2018, 26, 10109-10118. [CrossRef]

35. He, X.; Ning, T.; Pei, L.; Zheng, J.; Li, J.; Wen, X.D. Tunable hybridization of graphene plasmons and dielectric modes for highly confined light transmit at terahertz wavelength. Opt. Express 2019, 27, 5961-5972. [CrossRef]

36. Ye, L.; Sui, K.; Liu, Y.; Zhang, M.; Liu, Q. Graphene-based hybrid plasmonic waveguide for highly efficient broadband mid-infrared propagation and modulation. Opt. Express 2018, 26, 15935-15947. [CrossRef] [PubMed]

37. Gao, Y.; Ren, G.; Zhu, B.; Liu, H.; Lian, Y.; Jian, S. Analytical model for plasmon modes in graphene-coated nanowire. Opt. Express 2014, 22, 24322. [CrossRef]

38. Gao, Y.; Shadrivov, I.V. Second harmonic generation in graphene-coated nanowires. Opt. Lett. 2016, 41, 3623-3626. [CrossRef]

39. Hajati, M.; Hajati, Y. High-performance and low-loss plasmon waveguiding in graphene-coated nanowire with substrate. J. Opt. Soc. Am. B 2016, 33, 2560-2565. [CrossRef]

40. Teng, D.; Wang, K.; Li, Z.; Zhao, Y. Graphene-coated nanowire dimers for deep subwavelength waveguiding in mid-infrared range. Opt. Express 2019, 27, 12458-12469. [CrossRef]

41. Davoyan, A.R.; Engheta, N. Salient Features of Deeply Subwavelength Guiding of Terahertz Radiation in Graphene-Coated Fibers. ACS Photonics 2016, 3, 737-742. [CrossRef]

42. Liang, H.; Zhang, L.; Zhang, S.; Cao, T.; Alù, A.; Ruan, S.; Qiu, C.W. Gate-Programmable Electro-Optical Addressing Array of Graphene-Coated Nanowires with Sub-10 nm Resolution. ACS Photonics 2016, 3, 1847-1853. [CrossRef]

43. Kuzmin, D.A.; Bychkov, I.V.; Shavrov, V.G.; Temnov, V.V.; Lee, H.I.; Mok, J. Plasmonically induced magnetic field in graphene-coated nanowires. Opt. Lett. 2016, 41, 396-399. [CrossRef] [PubMed]

44. Huang, Y.; Zhang, L.; Yin, H.; Zhang, M.; Su, H.; Li, I.; Liang, H. Graphene-coated nanowires with a drop-shaped cross section for $10 \mathrm{~nm}$ confinement and $1 \mathrm{~mm}$ propagation. Opt. Lett. 2017, 42, 2078-2081. [CrossRef] [PubMed]

45. Zhang, T.; Chen, L.; Li, X. Graphene-based tunable broadband hyperlens for far-field subdiffraction imaging at mid-infrared frequencies. Opt. Express 2013, 21, 20888-20899. [CrossRef] [PubMed]

46. Nikitin, A.Y.; Guinea, F.; Garcia-Vidal, F.J.; Martin-Moreno, L. Fields radiated by a nanoemitter in a graphene sheet. Phys. Rev. B 2011, 84, 195446. [CrossRef]

47. Francescato, Y.; Giannini, V.; Maier, S.A. Strongly confined gap plasmon modes in graphene sandwiches and graphene-on-silicon. New J. Phys. 2013, 15, 063020. [CrossRef]

48. Zhou, Y.; Zhu, Y.Y.; Zhang, K.; Wu, H.W.; Peng, R.W.; Fan, R.H.; Wang, M. Plasmonic band structures in doped graphene tubes. Opt. Express 2017, 25, 12081-12089. [CrossRef] [PubMed]

49. Hanson, G.W. Quasi-transverse electromagnetic modes supported by a graphene parallel-plate waveguide. J. Appl. Phys. 2008, 104, 084314. [CrossRef]

50. Xu, J.; Shi, N.; Chen, Y.; Lu, X.; Wei, H.; Lu, Y.; Liu, N.; Zhang, B.; Wang, J. TM $\mathrm{TM}_{01}$ mode in a cylindrical hybrid plasmonic waveguide with large propagation length. Appl. Opt. 2018, 57, 4043-4047. [CrossRef] [PubMed]

51. Oulton, R.F.; Sorger, V.J.; Pile, D.F.P.; Genov, D.A.; Zhang, X. A hybrid plasmonic waveguide for sub-wavelength confinement and long range propagation. Nat. Photonics 2008, 2, 496-500. [CrossRef]

52. Ghosh, R.R.; Thomas, A.; Bhardwaj, P.; Subramanian, S.; Ahmed, K.; Dhawan, A. Design of long-range hybrid plasmonic waveguides. Opt. Compon. Mater. XVI 2019, 10914, $109141 \mathrm{Z}$.

53. Berini, P. Figures of merit for surface plasmon waveguides. Opt. Express 2006, 14, 13030-13042. [CrossRef] [PubMed]

(C) 2019 by the authors. Licensee MDPI, Basel, Switzerland. This article is an open access article distributed under the terms and conditions of the Creative Commons Attribution (CC BY) license (http://creativecommons.org/licenses/by/4.0/). 